Acta Univ. Sapientiae, Informatica 11, 1 (2019) 65-79

DOI: 10.2478 /ausi-2019-0005

\title{
Automatic detection of hard and soft exudates from retinal fundus images
}

\author{
Bálint BORSOS \\ Sapientia Hungarian University of Transylvania, \\ Cluj-Napoca, Romania \\ Dept. of Electrical Engineering, Târgu Mureş \\ email: bborsos55@gmail.com \\ László NAGY \\ Óbuda University, Budapest, Hungary \\ University Research, Innovation and Service Center \\ email: lnagy.priv@gmail.com

\section{David ICLĂNZAN} \\ Sapientia Hungarian University of Transylvania, \\ Cluj-Napoca, Romania \\ Dept. of Mathematics-Informatics, Târgu Mureş \\ email: iclanzan@ms.sapientia.ro

\section{László SZILÁGYI} \\ Sapientia Hungarian University of Transylvania, \\ Cluj-Napoca, Romania \\ Dept. of Electrical Engineering, Târgu Mureş \\ Óbuda University, Budapest, Hungary \\ University Research, Innovation and Service Center \\ email: lalo@ms.sapientia.ro
}

Computing Classification System 1998: I.2.1

Mathematics Subject Classification 2010: $68 \mathrm{~T} 10$

Key words and phrases: image segmentation, diabetic retinopathy, exudate detection 


\begin{abstract}
According to WHO estimates, 400 million people suffer from diabetes, and this number is likely to double by year 2030. Unfortunately, diabetes can have severe complications like glaucoma or retinopathy, which both can cause blindness. The main goal of our research is to provide an automated procedure that can detect retinopathy-related lesions of the retina from fundus images. This paper focuses on the segmentation of so-called white lesions of the retina that include hard and soft exudates. The established procedure consists of three main phases. The preprocessing step compensates the various luminosity patterns found in retinal images, using background and foreground pixel extraction and a data normalization operator similar to Z-transform. This is followed by a modified SLIC algorithm that provides homogeneous superpixels in the image. The final step is an ANN-based classification of pixels using fifteen features extracted from the neighborhood of the pixels taken from the equalized images and from the properties of the superpixel where the pixel belongs. The proposed methodology was tested using high-resolution fundus images originating from the IDRiD database. Pixelwise accuracy is characterized by a $54 \%$ Dice score in average, but the presence of exudates is detected with $94 \%$ precision.
\end{abstract}

\title{
1 Introduction
}

Retinopathy is a severe complication of diabetes, which can lead to partial or total loss of sight. Several million people are affected by retinopathy of various grades [14]. Retinopathy can be diagnosed via analysing the image of the retina, which is usually acquired with a fundus camera. In the clinical practice of developing countries, fundus images are recorded in certain regional hospitals, but they are sent to a central hospital for evaluation by a qualified human expert. In order to build a screening for mass population, it would be necessary to purchase more fundus cameras and train lots of humans to become qualified experts. While the first condition can be fulfilled by raising funds, the second condition regarding human experts is a more difficult one. This is why there is a strong need for well trained computer systems that can reliably separate obvious negative cases from suspected positive ones, and draw the attention of the human experts to the latter. This way it is possible to create screening systems without needing lots of more human experts.

Retinopathy can cause various lesions on the retina. Some examples are shown in Fig. 1. Microaneurysms and hemorrhages are collectively called red lesions, while hard and soft exudates together are referred to as white lesions. Although retinopathy is usually manifested with all these lesion types, just 


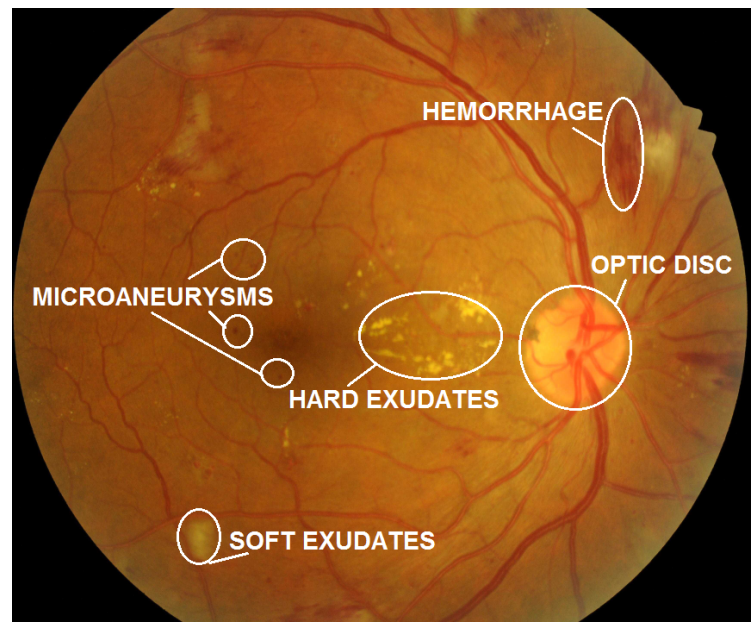

Figure 1: Retinal fundus image, indicating the anatomical parts relevant from the point of view of this study, and showing examples for the four types of the lesions. The original image was taken from the IDRid database [18].

as in the case shown in Fig. 1, the most studies from the literature focus on the detection or segmentation of only the red or the white lesions. To comply with this trend, we chose to dedicate the current study to the white lesion segmentation problem.

The computerized analysis of fundus images has been investigated for over two decades, and this process does not seem to slow down at all. The "Diabetic Retinopathy: Segmentation and Grading Challenge" [18] organized jointly with the IEEE International Symposium on Biomedical Imaging (ISBI 2018), with its high resolution retinal fundus image database, even seems to have an intensifying effect upon this research branch.

There are a few review articles in the field (e.g. $[9,16])$, which give us a concise insight into the exudate detection problem. The methodology includes solutions based on: global and adaptive thresholding [19, 10], region growing [15], clustering in color space [7], morphological operations [17, 23], edge detection and mixture modeling $[4,5,3]$, active contours and Naïve Bayes classifier [8], various supervised classifiers in competition [21], support vector machine [20,6], perceptron network combined with graph-cut algorithm [13], circular Hough transform combined with CNN networks [2], and CNN with deep learning $[11,12]$. 
This paper proposes a multi-step procedure for the exudate detection and segmentation problem, consisting of image intensity compensation, superpixel extraction, and supervised classification of pixels using a perceptron network, based on 15 features extracted from the neighborhood of the pixels and the superpixels they belong to. The proposed method will be evaluated using images from the IDRiD dataset [18].

The rest of this paper is structured as follows: Section 2 gives details on the proposed methodology. Section 3 exhibits and discusses the achieved results. Finally, Section 4 concludes the investigation.

\section{Materials and methods}

\subsection{Data sets}

This study relies on a subset of the Indian Diabetic Retinopathy Image Dataset (IDRiD) [18]. IDRiD contains 50 annotated positive images with soft and/or hard exudates, for which the optic disc mask is also available. Further 89 negative images were involved in the study, for these images we have produced optic disc masks. All these images were acquired using a Kowa VX-10 alpha digital fundus camera. Each positive image is accompanied by two masks that indicate the position of hard and soft exudates separately. We considered that the images in IDRiD have too high resolution $(4288 \times 2848$ pixels $)$ and too few components kept during JPEG encoding, so we resampled all retinal images and masks to $1072 \times 712$ pixels before proceeding to any processing step.

\subsection{Data processing}

The multi-step procedure proposed in this paper translates the image segmentation task into a classification problem, and provides a machine learning solution based on artificial neural networks (ANN) to complete the job. The classification takes place at the level of pixels. So each pixel is provided a feature vector, which includes properties of the neighborhood of the pixels, and properties of the superpixel it belongs to. This way the procedure needs to have the following steps (see Fig. 2): intensity compensation, superpixel generation, feature extraction, ANN training, ANN testing (prediction with ANN), and statistical evaluation. 


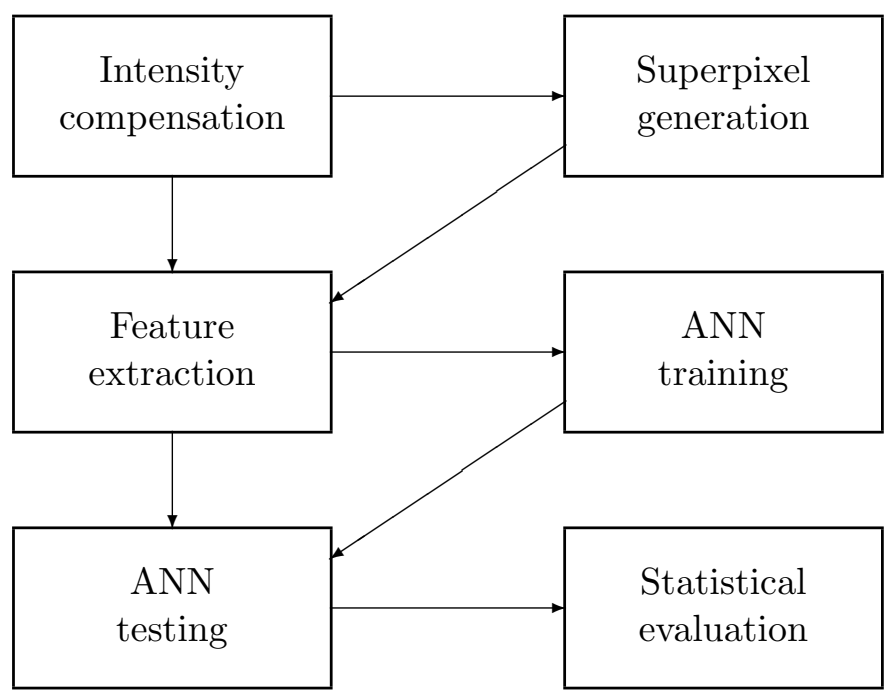

Figure 2: Block diagram of the proposed method.

\subsection{Intensity compensation}

A full processing of fundus images should be able to distinguish all anatomical structures and all lesions from the background. The ideal would be to have a constant background color. Unfortunately, the reality is usually very far from the ideal case. Some parts of most recorded fundus images appear darker than the others. This is why it is necessary to design a compensation scheme that would not affect the anatomical parts [22].

Our intensity compensation method is inspired from the work of Sánchez et al. [19]. First, we estimate which are the background pixel, and then we apply intensity correction based on the local statistics, average and standard deviation (STD) of intensities. Since the anatomical parts have significantly different color compared to their immediate neighborhood (background) and their edges are usually sharp, they are likely to be detected via thresholding. We performed a blur filter with mask sizes $39 \times 39$ pixels, and compared the blurred image from the original one. Wherever the absolute luminosity difference exceeds the half of the global intensity STD, those areas are declared anatomical parts. All other pixels are considered background pixels and they participate in the compensation process. At least $80 \%$ of the pixels belong to the background in most fundus images. 
To produce a compensation of intensities based on background pixels, it is necessary to extract the local average and STD of intensities for every background pixels. Since this would be a very time consuming process, we adapted the recipe given in [19], and turned to an approximation scheme. The whole fundus image was divided into $4 \times 4=16$ equal rectangles, which in our case contained $268 \times 178$ pixels each. The average and STD intensity of background pixels situated in each rectangle $\left(\mu_{i}\right.$ and $\left.\sigma_{i}, i=1 \ldots 16\right)$ was then computed. Finally, the approximated average and STD intensity for each pixel (not only background ones) was interpolated from the values obtained in the four (or less in the proximity of margins) such rectangles, using as weighting coefficient the -1 power of the physical distance between the pixel and the center of the rectangle. This way the difference and STD values of neighbor pixels were found quite similar, which conforms to our previous expectation that the bias field varies smoothly along the original image.

The compensation of any pixel situated at coordinates $(x, y)$ is finally performed with the formula:

$$
\widetilde{I}(x, y)=\frac{I(x, y)-\hat{\mu}_{\text {neigh }}(x, y)}{\bar{\sigma}_{\text {neigh }}(x, y)}+\mu_{\text {global }}
$$

with

$$
\bar{\sigma}_{\text {neigh }}(x, y)=\frac{\widehat{\sigma}_{\text {neigh }}(x, y)}{\frac{1}{16} \sum_{i=1}^{16} \sigma_{i}},
$$

where $\hat{\mu}_{\text {neigh }}(x, y)$ and $\hat{\sigma}_{\text {neigh }}(x, y)$ represent the interpolated average and STD intensity at pixel $(x, y)$, and $\mu_{\text {global }}$ is the desired average intensity of the compensated images, which can be a freely chosen value.

\subsection{Superpixel generation}

The images with compensated intensity obtained in the previous section were fed to a procedure that identified homogeneous spots or superpixels in them. The procedure was based on the so-called simple linear iterative clustering (SLIC) [1] algorithm. The original SLIC uses the k-means algorithm to cluster pixels using a composite distance function that includes components of physical distance and color difference. To assure the high speed of superpixel creation, when pixels are assigned to the closest cluster, only those cluster propotypes are tested which are situated within a short distance from the given pixel, as distant ones have no chance to be the closest according to the composite difference criterion. 
SLIC offers the chance for the user to set the approximate size of clusters. As cluster centers are initially sampled on a regular grid Q pixels apart, the approximate number of pixels belonging to each cluster will be $\mathrm{Q}^{2}$. In our implementation, the value of $\mathrm{Q}$ was set to 9 , and the composite distance between two pixels at coordinates $\left(x_{1}, y_{1}\right)$ and $\left(x_{2}, y_{2}\right)$, having gray intensities $g_{1}$ and $\mathrm{g}_{2}$, respectively, was considered as

$$
d=\sqrt{\left(x_{1}-x_{2}\right)^{2}+\left(y_{1}-y_{2}\right)^{2}+\frac{1}{5}\left(g_{1}-g_{2}\right)^{2}} .
$$

With these settings, SLIC provided approximately 6300 superpixels in each image. As a further step, all such superpixels or clusters were analyzed from the point of view of their intensity distribution. Those clusters in which the standard deviation exceeded a predefined threshold value $\theta$ were further separated into two clusters using the k-means algorithm. In this case only the pixel intensities were considered and not their position within the cluster. The value of the threshold $\theta$ was chosen such a way, that $5-10 \%$ of the clusters would be further separated into two parts. The value was chosen as $\theta=7.7$ units on the $0 \ldots 255$ gray scale. Finally, the number of superpixels or clusters was close to 7000 in each image.

\subsection{Feature generation}

Pixels of the retina images were going to be classified using a supervised machine learning approach. A feature vector was extracted for each pixel of the 50 retina images, with the following composition:

- 1-3: pixel intensity in red, green, and blue channels;

- 4-9: minimum, maximum, and average of green channel intensities extracted from $5 \times 5$ and $11 \times 11$ sized neighborhood of the pixel;

- 10: distance of the pixel from the closest point of the optic disc;

- 11: size of the superpixels where the pixel belongs;

- 12-15: average value in green channel, and the 10, 50, and 90 percentile values of green channel intensity within the superpixel where the pixel belongs.

These fifteen features characterize each pixel of the retina images. In the next section we will attempt to distinguish exudates pixels from normal ones using an artificial neural network based approach. 


\subsection{ANN training and testing}

An artificial neural network (ANN) was trained to separate exudates from other pixels of the retina images. The perceptron network employed for this problem consisted of four layers. The input layer has a dedicated neuron for each of the fifteen features listed in the previous subsection, the two hidden layers consists of seven neurons each, while there is a single neuron in the output layer.

To train the neural network, the "leave one out" technique was employed, namely we trained an ANN for each of the 50 retina images, using randomly selected pixels of the other 49 images as training data and their labeling as expected values. Train data for each network consisted of 4900 negative pixels, 600 hard exudate pixels and 500 soft exudate pixels.

The ANN deployed for hard and soft exudate detection was the one implemented in OpenCV ver. 3.1.0.

\subsection{Evaluation criteria}

Section 3 will provide a statistical evaluation of the obtained results. Pixelwise evaluation is performed based on the number of true positives (TP), false negatives (FN), false positives (FP) and true negatives (TN), and the following accuracy indicators will be computed:

- Sensitivity or true positive rate (TPR), defined as TPR $=\frac{T P}{\mathrm{TP}+\mathrm{FN}}$;

- Specificity or true negative rate $(\mathrm{TNR})$, defined as $\mathrm{TNR}=\frac{\mathrm{TN}}{\mathrm{TN}+\mathrm{FP}}$;

- Dice score (DS), defined as DS $=\frac{2 \times \mathrm{TP}}{2 \times \mathrm{TP}+\mathrm{FN}+\mathrm{FP}}$.

These statistical indicators will be analyzed separately in images with various amounts of exudate pixels.

\section{Results and discussion}

All 50 retina images with positive (exudate) pixels, and all 89 negative images underwent the above described processing steps. Figure 3 presents the intermediary results obtained for one of the images, starting from the original

color image and its green channel, the region of interest consisting from the whole retinal part of the image with the optic disc removed, the estimated set of background pixels, the intensity compensated image and the superpixels 
(a)

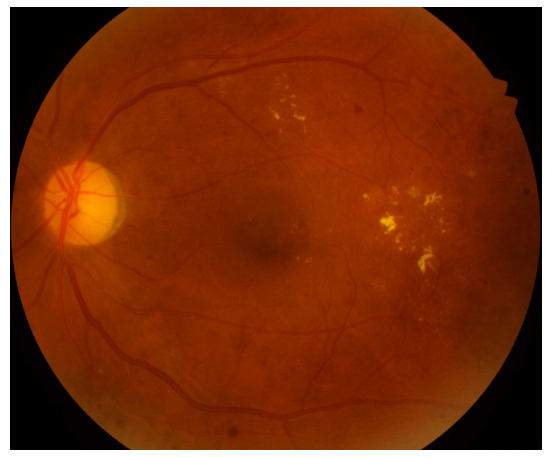

(c)

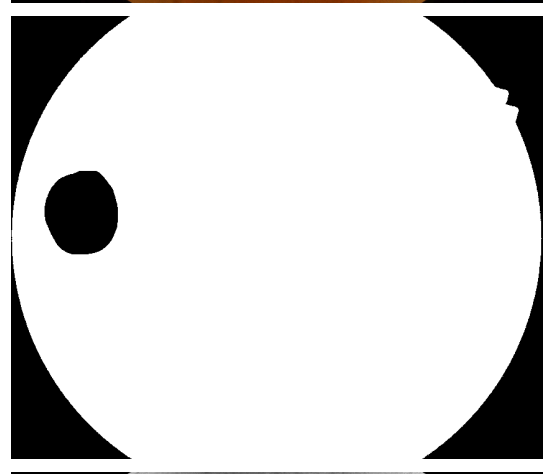

(e)

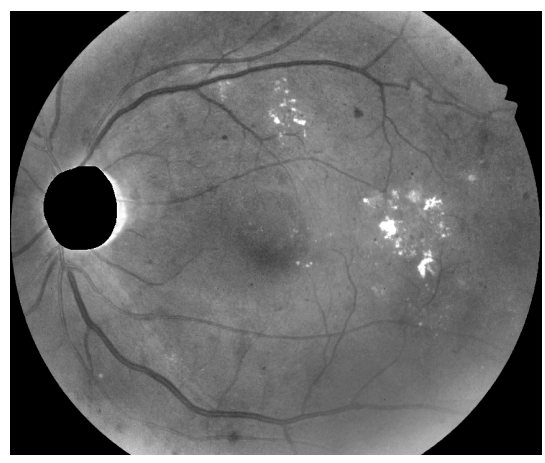

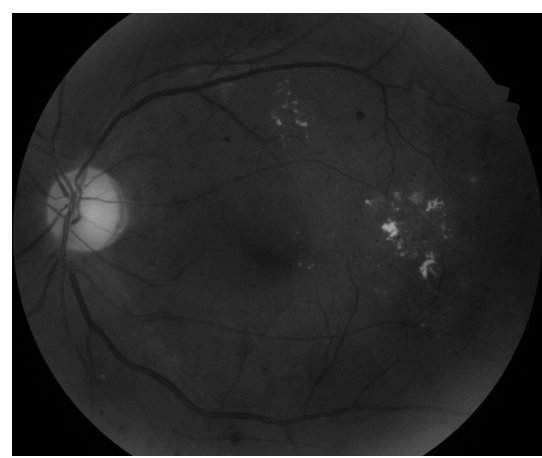

(b)

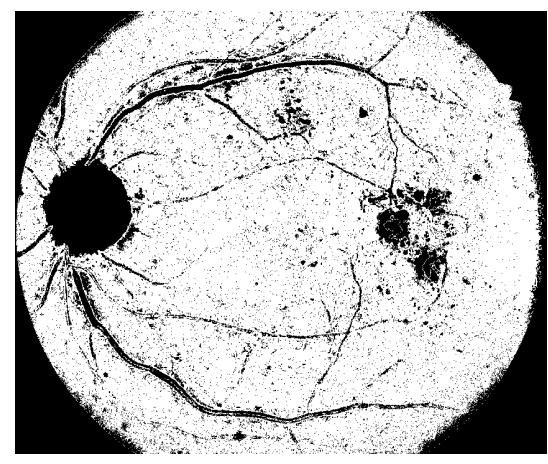

(d)

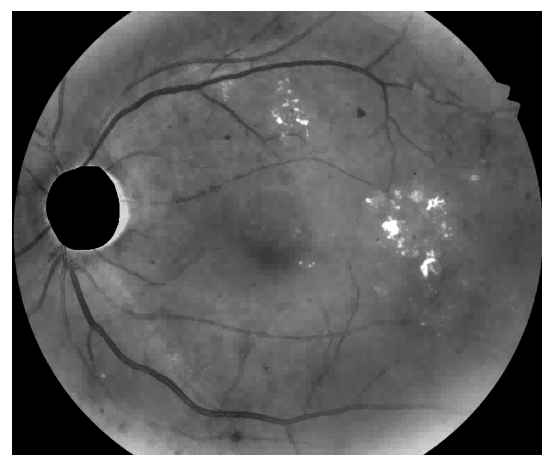

(f)

Figure 3: Intermediary results of the exudate detection procedure: (a) original color image; (b) the green channel of the original image; (c) region of interest: the whole retina image without the area of the optic disc; (d) the background pixels detected by the intensity compensation method; (e) the intensity compensated image; (f) the superpixels found by the modified SLIC algorithm. 
(a)
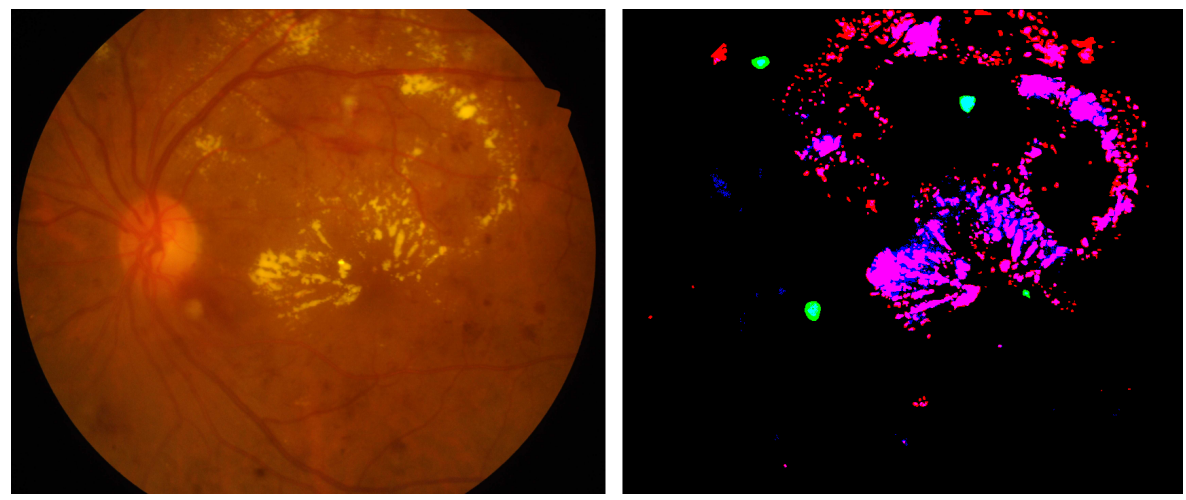

(b)
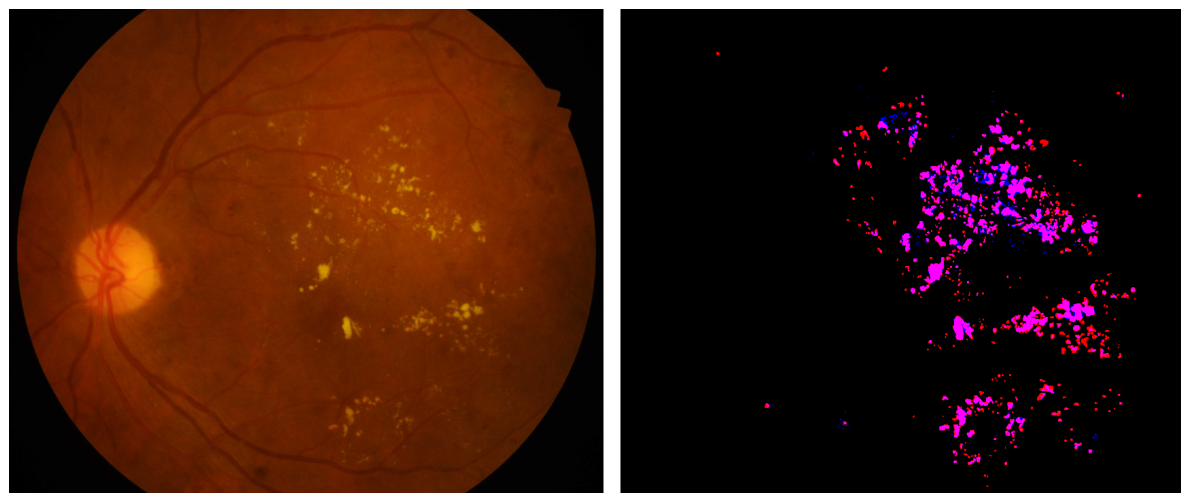

(c)

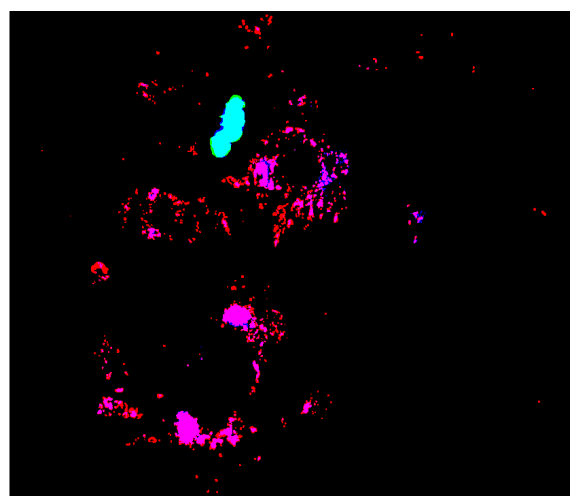

Figure 4: Three of the input retina images and the detected exudates: magenta and cyan represent detected hard and soft exudates, respectively; red and green indicate undetected hard and soft exudates, respectively; false positives are shown in blue. 

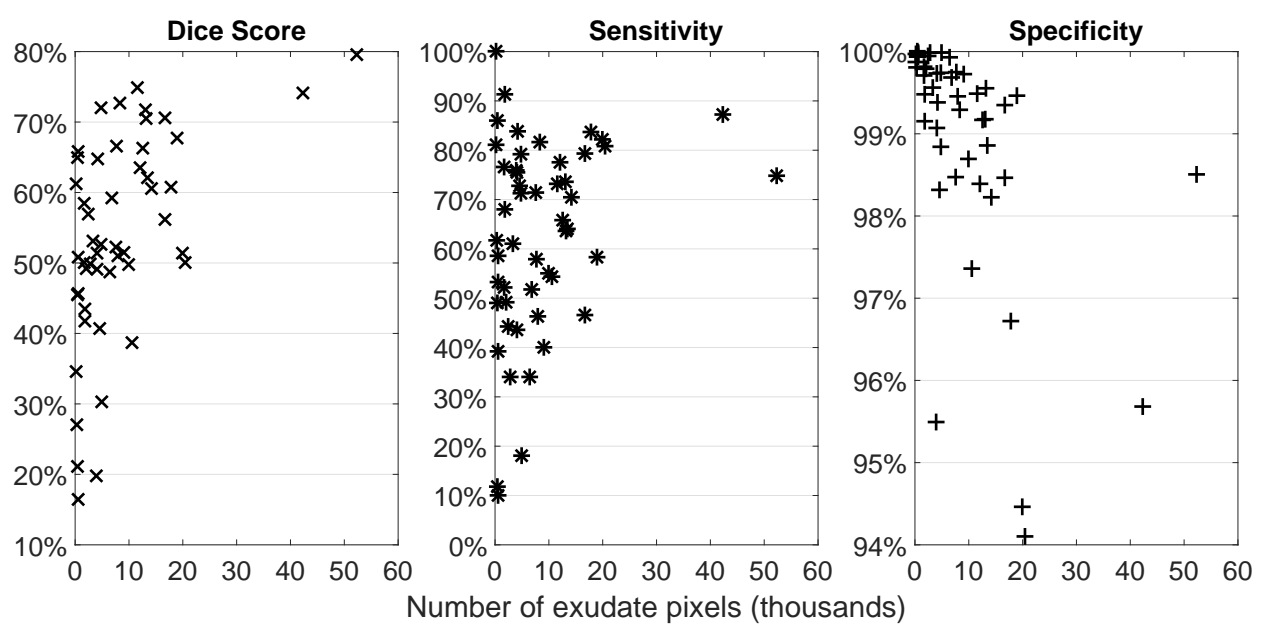

Figure 5: Segmentation quality indicators obtained for individual retina images, plotted against the actual number of exudate pixels in the image according to the ground truth.

identified in it. The feature vector was extracted for all pixels of all images involved in this study.

For each image with exudate pixels, a dedicated ANN was trained to obtain the segmentation, as described in Section 2.6. For the negative images, a single ANN was trained using pixels from all 50 images that contain positives. Detailed results of the segmentation are presented in the following.

Figure 4 presents the segmentation outcome for three images with positives. The left column shows the original color images, while the right column indicates the found and missed structures. The red, green and blue channels of the color image are used according to the following logic:

- red channel is set to maximum intensity wherever there is a hard exudate pixel according to the ground truth;

- green channel is set to maximum intensity wherever there is a soft exudate pixel according to the ground truth;

- blue channel is set to maximum intensity wherever the ANN estimates that there is an exudate pixel;

- all other pixels in all color channels are set to zero intensity. 


\begin{tabular}{l|cccc|c}
\hline \multirow{2}{*}{$\begin{array}{l}\text { Accuracy } \\
\text { indicators }\end{array}$} & \multicolumn{4}{|c|}{ Cases with exudate pixel count } & Average of \\
\cline { 2 - 5 } & $\geq 30000$ & $\geq 10000$ & $\geq 3000$ & $\geq 1000$ & all cases \\
\hline Dice Score & $76.83 \%$ & $63.67 \%$ & $57.85 \%$ & $56.35 \%$ & $53.75 \%$ \\
Sensitivity & $80.99 \%$ & $70.96 \%$ & $65.43 \%$ & $64.26 \%$ & $62.42 \%$ \\
Specificity & $97.09 \%$ & $97.94 \%$ & $98.52 \%$ & $98.75 \%$ & $98.99 \%$ \\
\hline
\end{tabular}

Table 1: Average values of the accuracy indicators for various subsets of the input images, defined in accordance to the actual number of exudate pixels in the images.

This notation means that correct decisions are denoted by magenta (identified hard exudates), cyan (identified soft exudates), and black (true negatives), while red (missed hard exudates), green (missed soft exudates), and blue (false positives) indicate wrong decisions. Larger spots of hard exudates are usually found, while smaller ones are more likely to be missed.

Figure 5 shows the main statistical quality indicator values obtained for individual retina images plotted against the number of actual exudate pixels present in the images. This figure shows that Dice Score and Sensitivity is generally higher in the segmentation outcome of images with larger number of exudate pixels. The variation os Specificity is apparently reversed.

Table 1 indicates the average values of main statistical quality indicators obtained in various subsets of the images that contain positive pixels. Subsets restrict the whole set of positive images to those, which contain at least a certain threshold number of positive pixels, the threshold varying from 1000 to 30000. As it is expected, images with more positive pixels are processed with better accuracy, at least from the point of view of Dice Score and Sensitivity.

If we consider the pixels of the whole set of positive images with their segmentation outcome, and compute the overall accuracy indicators for this whole set, we obtain DS $=60.52 \%$, TPR $=68.98 \%$, and TNR $=99.01 \%$.

For the negative images it only makes sense to extract the Specificity value, which showed and average of $98.63 \%$. For further development, it would be necessary to propose an additional processing step to suppress the false positives. 


\section{Conclusion}

This paper proposed an image segmentation procedure to identify the presence of hard and soft exudates in retinal fundus images. The proposed method was tested on 50 positive and 89 negative cases taken from the IDRiD database. The sensitivity values above $1 / 3$, which we consider enough to detect the presence of exudates, was found in $94 \%$ of the positive cases, making the proposed method a good candidate for integration into a future screening application.

\section{Acknowledgements}

The work of B. Borsos was funded by the Szekely Forerunner Federation. The work of L. Szilágyi was supported by the ÚNKP 18-4 New National Excellence Program of the Ministry of Human Capacities of Hungary, contract no. OE-OF, 325/6, 2018. L. Szilágyi is Bolyai Research Fellow of the Hungarian Academy of Sciences.

\section{References}

[1] R. Achanta, A. Shaji, K. Smith, A. Lucchi, P. Fua, S. Süsstrunk, SLIC superpixels compared to state-of-the-art superpixel methods, IEEE Trans. PAMI 34 (2012) 2274-2282. $\Rightarrow 70$

[2] K. Adem, Exudate detection for diabetic retinopathy with circular Hough transformation and convolutional neural networks, Expert Syst. Appl. 114 (2018) 289-295. $\Rightarrow 67$

[3] C. Agurto, V. Murray, H. Yu, J. Wigdahl, M. Pattichis, S. Nemeth, S. Barriga, P. Soliz, A multiscale optimization approach to detect exudates in the macula, IEEE J. Biomed. Health Inf. 18, 4 (2014) 1328-1337. $\Rightarrow 67$

[4] K. S. Deepak, J. Sivaswamy, Automatic assessment of macular edema from color retinal images, IEEE Trans. Med. Imag. 31, 3 (2012) 766-776. $\Rightarrow 67$

[5] M. Esmaeili, H. Rabbani, A. M. Dehnavi, A. Dehghani, Automatic detection of exudates and optic disc in retinal images using curvelet transform, IET Image Proc. 6 (2012) 1005-1013. $\Rightarrow 67$

[6] L. Giancardo, F. Meriaudeau, T. P. Karnowski, Y. Q. Li, S. Garg, K. W. Tobin Jr., E. Chaum, Exudate-based diabetic macular edema detection in fundus images using publicly available datasets, Med. Image Anal. 16, 1 (2012) 216-226. $\Rightarrow 67$

[7] C. E. Hann, J. A. Revie, D. Hewett, J. G. Chase, G. M. Shaw, Screening for diabetic retinopathy using computer vision and physiological markers, J. Diabetes Sci. Technol. 3, 4 (2009) 819-834. $\Rightarrow 67$ 
[8] B. Harangi, A. Hajdú, Automatic exudate detection by fusing multiple active contours and regionwise classification, Comput. Biol. Med. 54 (2014) 156-171. $\Rightarrow 67$

[9] S. Joshi, P. T. Kerule, A review on exudates detection methods for diabetic retinopathy, Biomed. Pharmacoter. 97 (2018) 1454-1460. $\Rightarrow 67$

[10] J. Kaur, D. Mittal, A generalized method for the segmentation of exudates from pathological retinal fundus images, Biocybern. Biomed. Eng. 38, 1 (2018) 27-53. $\Rightarrow 67$

[11] P. Khojasteh, L. A. Passos Júnior, T. Carvalho, E. Rezende, B. Aliahmad, J. P. Papa, D. K. Kumar, Exudate detection in fundus images using deeply-learnable features, Comput. Biol. Med. 104 (2019) 62-69. $\Rightarrow 67$

[12] P. Khojasteh, B. Aliahmad, D. K. Kumar, A novel color space of fundus images for automatic exudates detection, Biomed. Sign. Proc. Control 49 (2019) 240 249. $\Rightarrow 67$

[13] W. Kusakunniran, Q. Wu, P. Ritthipravat, J. Zhang, Hard exudates segmentation based on learned initial seeds and iterative graph cut, Comput. Meth. Prog. Biol. 158 (2018) 173-183. $\Rightarrow 67$

[14] J. L. Leasher, R. R. Bourne, S. R. Flaxman, J. B. Jonas, J. Keeffe, K. Naidoo, K. Pesudovs, H. Price, R. A. White, T. Y. Wong, S. Resnikoff, H. R. Taylor, et al., Global estimates on the number of people blind or visually impaired by diabetic retinopathy: a meta-analysis from 1990-2010, Diabetes Care 39 (2016) 1643-1649. $\Rightarrow 66$

[15] J. Lowell, A. Hunter, D. Steel, A. Basu, R. Ryder, E. Fletcher, L. Kennedy, Optic nerve head segmentation, IEEE Trans. Med. Imag. 23, 2 (2005) 256-264. $\Rightarrow 67$

[16] M. R. K. Mookiah, U. R. Acharya, C. K. Chua, C. M. Lim, E. Y. K. Ng, A. Laude, Computer-aided diagnosis of diabetic retinopathy: a review, Comput. Biol. Med. 43 (2013) 2136-2155. $\Rightarrow 67$

[17] J. Nayak, P. S. Bhat, U. R. Acharya, C. Lim, M. Kagathi, Automated identification of different stages of diabetic retinopathy using digital fundus images, $J$. Med. Syst. 32 (2008) 107-115. $\Rightarrow 67$

[18] P. Porwal, S. Pachade, R. Kamble, M. Kokare, G. Deshmukh, V. Sahasrabuddhe, F. Meriaudeau, Indian Diabetic Retinopathy Image Dataset (IDRiD): A database for diabetic retinopathy screening research, Data $\mathbf{3}, 3$ (2018) 25 . $\Rightarrow$ 67,68

[19] C. I. Sánchez, M. García, A. Mayo, M. I. Lopez, R. Hornero, Retinal image analysis based on mixture models to detect hard exudates, Med. Image Anal. 13, 4 (2009) 650-658. $\Rightarrow 67,69,70$

[20] D. Sidibé, I. Sadek, F. Mériaudeau, Discrimination of retinal images containing bright lesions using sparse coded features and SVM, Comput. Biol. Med. 62 (2015) 175-184. $\Rightarrow 67$

[21] R. Sohini, P. Dara, K. K. Parhi, DREAM: diabetic retinopathy analysis using machine learning, IEEE J. Biomed. Health Inf. 18, 5 (2014) 1717-1729. $\Rightarrow 67$ 
[22] L. Szilágyi, S. M. Szilágyi, B. Benyó, Efficient inhomogeneity compensation using fuzzy c-means clustering models, Comput. Meth. Prog. Biol. 108 (2012) 80-89. $\Rightarrow 69$

[23] X. Zhang, G. Thibault, E. Decencière, B. Marcotegui, B. Laÿ, R. Danno, G. Cazuguel, G. Quellec, M. Lamard, P. Massin, A. Chabouis, Z. Victor, A. Erginay, Exudate detection in color retinal images for mass screening of diabetic retinopathy, Med. Image Anal. 18, 7 (2014) 1026-1043. $\Rightarrow 67$

Received: July 9, 2019・Revised: July 27, 2019 